\title{
Oblique Single-Cut Rotation Osteotomy for Correction of Femoral Varus-Torsional Deformities in 3D-Reconstructed Canine Bone Models
}

\author{
Hyeon-Ho Kim ${ }^{\dagger}$, Yoon-Ho Roh ${ }^{\dagger}$, Je-Hun Lee, Jae-Min Jeong, Seong Mok Jeong and Hae Beom Lee ${ }^{1}$ \\ College of Veterinary Medicine, Chungnam National University, Daejeon 34134, Korea
}

(Received: March 27, 2020 / Revised: July 16, 2020 / Accepted: August 03, 2020)

\begin{abstract}
The purpose of this study was to report the reliability and validity of oblique single-cut rotation osteotomy (OSCRO) in 3D-reconstructed canine bone models with femoral varus and torsional deformities. A healthy adult male beagle was recruited to create a 3D bone model, and this bone model was modified by using a 3D program. Fifteen bone models were constructed for this study. OSCRO simulation was performed in accordance with the plan after printing using a 3D printing machine. The anatomical lateral distal femoral angle (aLDFA), anteversion angle (AA), anatomical caudo-distal femoral angle (aCdDFA), mechanical caudo-distal femoral angle (mCdDFA) and pre- and postoperative bone length were calculated. There were no significant differences between the target values and postoperative values. In addition, the difference between pre- and postoperative bone length was small $(p=0.001)$. Our findings suggest that OSCRO could be an effective surgical option for MPL with bone deformities in small-breed dogs that often undergo conventional distal femoral osteotomy.
\end{abstract}

Key words : bone deformity, oblique single-cut rotation osteotomy, medial patellar luxation, torsion, varus.

\section{Introduction}

Medial patellar luxation (MPL) is a common orthopedic problem in dogs. The etiology of MPL is not entirely understood (20). However, several investigations into its cause have suggested that malalignment of the quadriceps mechanism leads to patellar luxation $(12,14,17)$. Bone deformities that contribute to this malalignment have been reported, including coxa vara, femoral varus, external torsion of the femur, shallow femoral trochlear groove, deviation of the tibial crest and tibial valgus $(17,20,21)$. MPL is considered a mixture of these complex skeletal deformities rather than an isolated disease of the stifle (18).

Many surgical methods for the correction of MPL have been reported, and their common goal is to improve quadriceps alignment $(3,14,20)$. This has been accomplished through conventional surgical treatments, including trochleoplasty and tibial tuberosity transposition with soft tissue reconstruction $(2,9,14)$. However, a recent study reported that the rate of re-luxation following these conventional surgical methods was as high as $8 \%$ if femoral varus and torsion were uncorrected $(1,2,5)$. Etiologically, the correction of the bone deformities with the medial displacement of the quadriceps femoris muscle group is gaining more attention in treating MPL (4, $16,21)$. In several studies, the rate of re-luxation became significantly lower following distal femoral osteotomy (DFO) with conventional methods (14). DFO is a recent surgical method focused on the correction of excessive femoral varus and decreased anteversion angle. However, DFO has several dis-

These authors contributed equally to this work.

${ }^{1}$ Corresponding author.

E-mail : seatiger76@cnu.ac.kr advantages. In the open wedge method, the gap between the fragments of the femur leads to a prolonged healing time and fixation instability. The closed wedge method, on the other hand, requires two osteotomies that result in bone loss and a prolonged surgical time (4). In addition, DFO is commonly performed in two following steps, correcting varus and torsion.

In human medicine, many studies have reported the use of oblique single-cut rotation osteotomy (OSCRO) for the correction of both angular and torsional deformities $(12,13,15,16)$. OSCRO can correct angular and torsional deformities simultaneously in one step by simply rotating the distal bone segment about a line perpendicular to the cutting plane. The advantage of OSCRO is that it allows a large contact area between the bone fragments without bone loss with only a single osteotomy $(8,10,11)$.

Despite this advantage and the many reports in human medicine, only two studies of OSCRO have been reported for the correction of radial deformities in veterinary medicine $(8,10)$. To the author's knowledge, OSCRO for the correction of femoral varus-torsion deformities in MPL has not been described.

The purpose of this study was to describe the possibilities, advantages and disadvantages of the application of OSCRO in 3D-reconstructed canine bone models to correct MPL with bone deformities.

\section{Materials and Methods}

A healthy adult male beagle weighing $10.7 \mathrm{~kg}$ was used to create a 3D bone model and was selected as a baseline for the present study. No abnormalities were found via physical or orthopedic examination or radiography. This study was approved by Chungnam National University Animal Care and Use Committee (No. CNU-00838). Computed tomogra- 


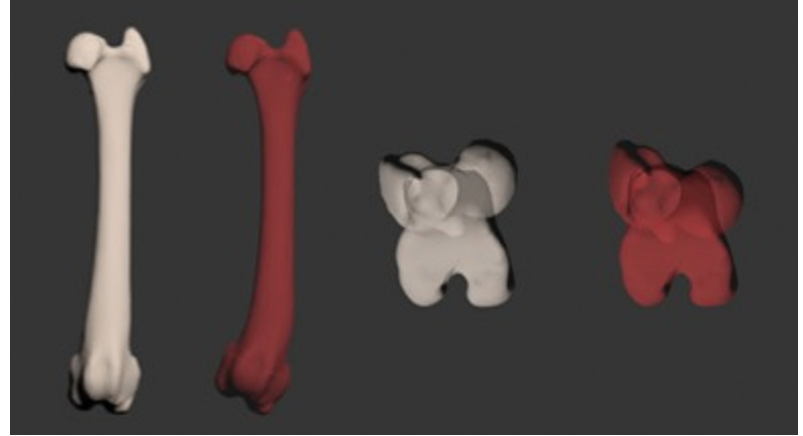

Fig 1. The normal bone model (white) was modified at 5-degree intervals. Varus and torsional deformities were created (red).

phy images of the dog were acquired with a 16-slice scanner (AlexionTM, Toshiba, Otawara, Japan). Images were obtained with a slice thickness of $1 \mathrm{~mm}$ and reconstructed as $3 \mathrm{D}$ images using image processing software. By using 3DS MAX software (3DS MAX, Autodesk, California, USA), the bone model was modified at the level of the distal one-third of the bone. The varus deformities were modified at 5-degree intervals (aLDFA: $100^{\circ}, 105^{\circ}$, and $110^{\circ}$ ), and torsional deformities were also modified at 5-degree intervals (anteversion angle: $0,5^{\circ}, 10^{\circ}, 15^{\circ}$, and $20^{\circ}$ ) (Fig 1). A total of 15 anomalies were reconstructed by combining the number of cases of each anomaly $(3 \times 5=15)$. Bone models were printed with 3D printer (Finbot-Z420, TPC mecatronics, Korea) and poly lactic acid filament (PLA filament, ESUN Inc, China).

\section{Surgical planning}

The surgical plan was performed based on a reported method (13). The angular component of the deformity (CORA, bisector line, deformity direction) was analyzed in the frontal view. The anatomical lateral distal femoral angle (aLDFA) and anteversion angle (AA) were measured. The magnitudes of the angulation $(\mathrm{A})$ and rotation $(\mathrm{R})$ of the deformities were calculated by the following equation: |Measured angle-
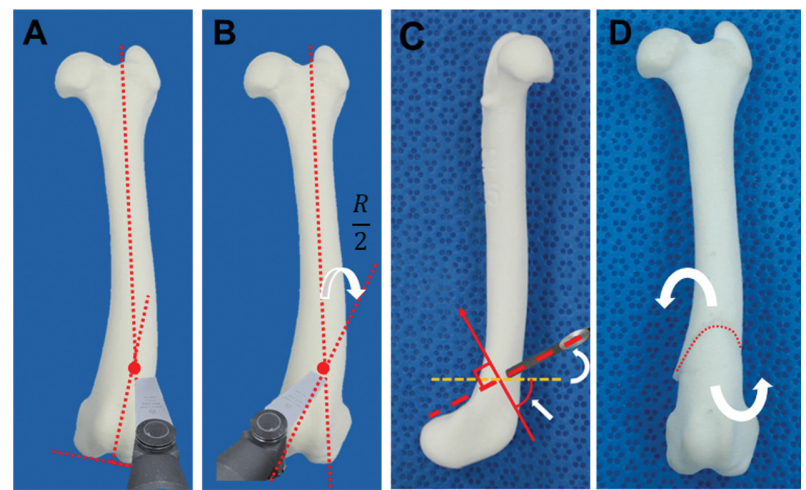

Fig 2. Photographs showing the surgical procedure. (A) The orientation of the axis for the correction was determined based on the CORA (red dot). (B) The axis was oriented by $R / 2$ in the opposite direction to the torsional deformity. (C) The axis was inclined longitudinally according to the inclined axis angle (white arrow). The osteotomy line was perpendicular to the new axis. (D) After osteotomy, the proximal segment of the femur was rotated around the new axis (black dot). target angle|. The orientation of the axis for the correction of angulation was determined (perpendicular to the plane of angulation; Fig 2A). Then, the axis was reoriented by $\mathrm{R} / 2$ in the opposite direction to the torsional deformity (Fig 2B) and inclined longitudinally by the amount calculated by the following equation (Fig 2C): inclined axis angle $=$ $\tan ^{-1}\left[\sin \left(\frac{\mathrm{R}}{2}\right) / \tan \left(\frac{\mathrm{A}}{2}\right)\right]$. After reorientation, the new axis line was on either the convex or concave side of the angular deformity. If the axis line was on the convex side, the axis was raised. In contrast, if the axis line was on the concave side, the axis was lowered. After completion of the osteotomy, the distal part of the bone was rotated along the axis perpendicular to the osteotomy line (Fig 2D). The actual magnitude of rotation along the axis was calculated by the following equation: actual rotation angle $=$ $\cos ^{-1}\left[\frac{1}{2}\{\cos (\mathrm{R})+\cos (\mathrm{A})+(\cos (\mathrm{R}) \times \cos (\mathrm{A})-1)\}\right]$.

\section{Outcome measures}

After rehearsal surgery, the photographs of bone model was taken with a camera (Canon EOS 750D, Canon Inc, Japan) projecting perpendicular to the bone model that lies next to the $25 \mathrm{~mm}$ radiographic reference ball. Values were measured using a radiographic software program (Viewrex ${ }^{\circledR}$, Techheim CO. LTD., Seoul, Korea). All the values for the femur were measured pre- and postoperatively using previously described methods (21). The anato mical lateral distal femoral angle (aLDFA) in the frontal view and the anteversion angle (AA) in the axis view were measured (Fig 3A and $3 B)$. To confirm the accuracy of the surgical method, the difference between the target angle and the postoperative angle was calculated as |Postoperative angle-Target angle|. The target angles were determined using the criteria from a previously reported study ( $\left.\mathrm{aLDFA}=95^{\circ}, \mathrm{AA}=25^{\circ}\right)(4)$. The anatomical caudo-distal femoral angle (aCdDFA) and mechanical caudo-distal femoral angle (mCdDFA) in the lateral view were measured (Fig 3C). To identify unintended changes
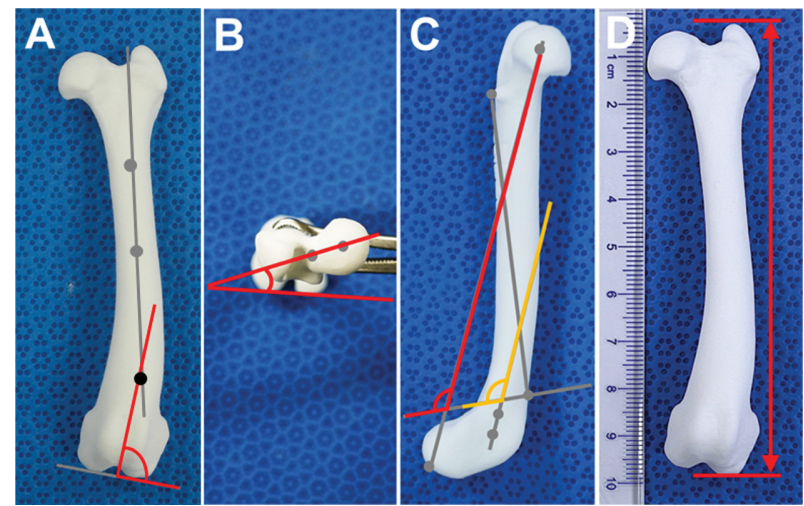

Fig 3. Photographs showing the measurements of the femur. (A) The center of the rotation angle (CORA, black dot) and anatomical lateral distal femoral angle (aLDFA, red line) were measured. (B) The anteversion angle (AA) was measured (red line). (C) The anatomical caudo-distal femoral angle (aCdDFA; yellow line) and mechanical caudo-distal femoral angle (mCdDFA; red line) were measured. (D) Length was measured. 
after surgery, the difference between the preoperative angle and the postoperative angle was calculated as |Postoperative angle - Preoperative angle|. The length of the bone in the frontal view was measured (Fig 3D). To identify changes after surgery, the difference between the preoperative length and postoperative length was calculated as |Postoperative length - Preoperative length|.

\section{Statistical analysis}

All data analysis was performed using a statistical software program (SPSS Statistics ver 22.0, IBM, New York, USA). Each outcome measured was expressed as the mean \pm SD. The normality of the data was tested with the Kolmogorov-Smirnov and Shapiro-Wilk tests. The difference between the target angle and postoperative angle (aLDFA and AA) was statistically evaluated with the independent samples t-test. The difference between the pre- and postoperative angles (aCdDFA and mCdDFA) was statistically evaluated with the paired t-test. The difference between the pre- and postoperative length was statistically evaluated with the Wilcoxon signed rank test.

\section{Results}

In this study, all bone models were constructed by the 3D model. There were 15 bone models with different aLDFAs and torsion. There were no significant differences between
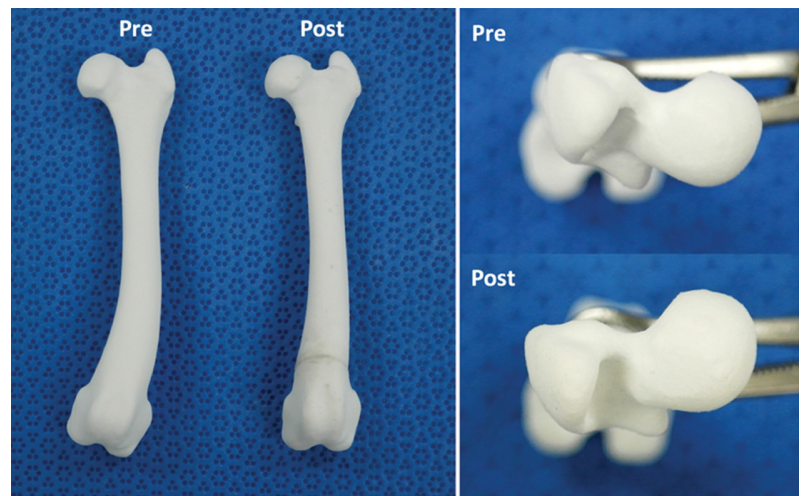

Fig 4. Photographs showing comparisons of the differences between the preoperative and postoperative bone models (frontal view: left, axis view: right). It was confirmed that the varus and torsional deformities were corrected. the measured data of all postoperative bone models, target values or preoperative values (Table 1). The mean postoperative aLDFA was $94.4^{\circ} \pm 1.2^{\circ}$. The range was from $92.9^{\circ}$ to $97.1^{\circ}$. There were no significant differences between the postoperative aLDFA and the target angle $(p=0.096$; Table $1)$. The mean postoperative $\mathrm{AA}$ was $25.5^{\circ} \pm 1.3^{\circ}$. The range was from $22.5^{\circ}$ to $27.1^{\circ}$. There were no significant differences between the postoperative AA and the target angle ( $p=$ 0.135 ; Fig 4 ). The difference between the preoperative aCdDFA and postoperative aCdDFA was $0^{\circ} \pm 2.4^{\circ}$. There were no significant differences between the preoperative aCdDFA and postoperative aCdDFA $(p=0.974)$. The difference between the preoperative mCdDFA and postoperative mCdDFA was $0.6^{\circ} \pm 1.4^{\circ}$. There were no significant differences between the preoperative mCdDFA and postoperative mCdDFA ( $p=0.123$; Fig 5$)$. The mean preoperative length was $9.4 \mathrm{~cm} \pm 0.6 \mathrm{~cm}$, and the mean postoperative length was $9.3 \mathrm{~cm} \pm 0.6 \mathrm{~cm}$. The difference between the preoperative

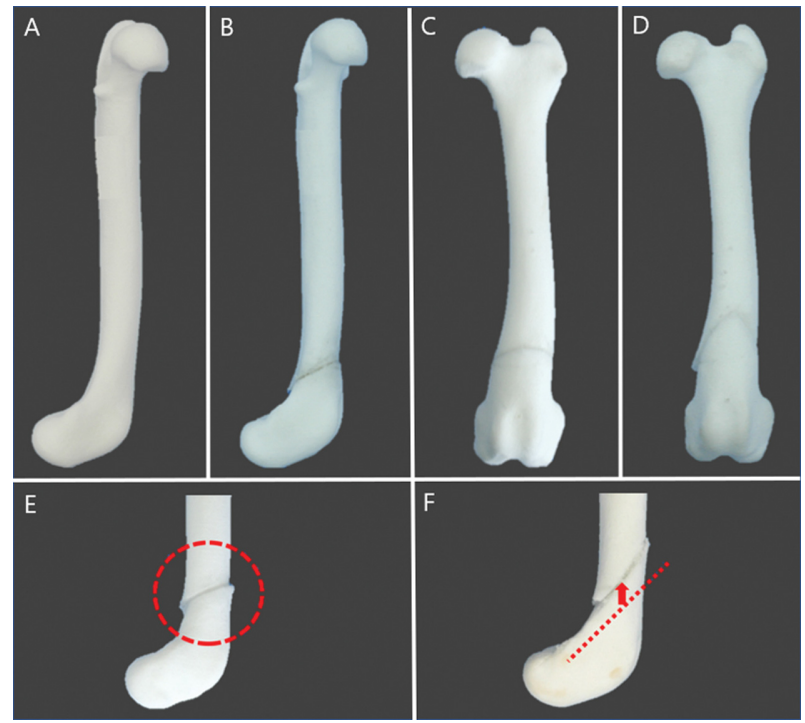

Fig 5. Photographs showing the preoperative bone model (A) and postoperative bone model $(\mathrm{B}, \mathrm{C}, \mathrm{D}, \mathrm{E}, \mathrm{F})$. On the medial view of the bone model (A) and (B), no significant difference was noted after the procedure. When comparing the translation of the distal bone segment postoperatively with the cranial view $(C, D)$ and medial view (E, F), no significant change was noted with the proximal modification of the osteotomy line (D, F) compared to the original osteotomy line $(\mathrm{C}, \mathrm{E})$.

Table 1. Comparing outcome variables after oblique single cut rotation osteotomy in the femur

\begin{tabular}{lcccc}
\hline \hline & Mean \pm SD $\left(^{\circ}\right)$ & $p$-Value & Target angle $\left(^{\circ}\right)$ & Range $\left.^{\circ}\right)$ \\
\hline Postoperative aLDFA $^{*}$ & $94.4 \pm 1.2$ & 0.096 & 95 & $92.9-97.1$ \\
Postoperative AA $^{*}$ & $25.5 \pm 1.3$ & 0.135 & 25 & $22.5-27.1$ \\
Postoperative aCdDFA - Preoperative aCdDFA & $0 \pm 2.4$ & 0.974 & - & - \\
Postoperative mCdDFA - Preoperative mCdDFA & $0.6 \pm 1.4$ & 0.123 & - & - \\
Preoperative length & $9.4 \pm 0.6$ & - & - & - \\
Postoperative length & $9.3 \pm 1.4$ & - & - & - \\
Postoperative length - Preoperative length & $-0.17 \pm 0.07$ & 0.001 & - & - \\
\hline
\end{tabular}

\footnotetext{
*Independent sample t-test, $p<0.05$.

Paired t-test, $p<0.05$.
}

Wilcoxon signed rank test, $p<0.05$. 
length and postoperative length was $-0.17 \mathrm{~cm} \pm 0.07 \mathrm{~cm}$. There were significant differences between the preoperative length and the postoperative length ( $p=0.001$; Fig 4$)$.

Of 15 stifles, 2 bone models had a translation after planned surgeries. The distal segment of the femur translocated cranially. Three models had osteotomy lines modified proximally due to the presence of sesamoid bone on the original osteotomy line.

\section{Discussion}

This study showed that OSCRO had great accuracy for the correction of angulation-torsion of the femur without any unintended deformities. There was no significant difference in accuracy between the plan and the postoperative results. The results of our study suggest that OSCRO is unlikely to affect unintended deformities; therefore, OSCRO might be an appropriate technique to correct severe MPL with bone deformities in the femur. Based on the ex vivo characteristics of this study, the application of these results to clinical cases seems promising.

The bone length was reduced after surgery, with a mean length of $1.7 \mathrm{~mm}$, which was $1.8 \%$ of the total bone. This likely results from two causes: first, procedural bone loss. The bone model utilized cutting with an oscillating saw (Synthes, DePuy Synthes, Massachusetts, USA). This saw blade has a thickness of approximately $0.6 \mathrm{~mm}$, which may have caused ablation by crushing and shearing the bone. Additionally, the imprecision of the cut due to the high torque may have also contributed to bone loss (19). The second cause may be the selection of the angulation correction axis (ACA). Because OSCRO is performed with vertically inclined osteotomy, many possible ACAs can be chosen (13). If the distal bone segment is rotated about the CORA on the transverse bisector line (tBL), no translational deformity occurs. However, as the ACA moves from the center to the concave side of the deformity along the $\mathrm{tBL}$, the bone length becomes shorter. As the ACA moves from the center to the convex side, the bone length becomes longer.

In this study, translation occurred in some models (Fig 5). However, there was little effect on the postoperative angle. The following reasons can be considered for this outcome. In other corrective osteotomies, translational deformities occur when the ACA does not correspond to the level of the transverse bisector line. In the case of OSCRO, since the surface of the osteotomy is ellipsoid, the osteotomy edges will translate to each other despite rotating around the center of the axis. Strictly, this is not the translation referred to above since no axis deviation occurs (13). Additionally, OSCRO has a wide range passing through the level of the CORA on the tBL because the osteotomy performed is vertically inclined. Therefore, even if the osteotomy line is modified, translation does not occur prominently when rotating around the planned axis (Fig 5).

The degree of inclination needed to perform osteotomy is not proportional to the magnitude of the angle but is determined by the ratio of angulation to the torsion angle. When the ratio of angulation to the torsion angle is 1 , the degree of inclination is $45^{\circ}$. If the ratio increases to greater than 1 , the degree of inclination increases by more than $45^{\circ}$. In contrast, if the ratio decreases to below 1 , the degree of inclination decreases to less than $45^{\circ}$. In the case of OSCRO for the correction of MPL a steep osteotomy line should be modified proximally because of the osteotomy direction towards anatomical structures such as the sesamoid bone, the femoral condyle and the insertion of the gastrocnemius muscle. In this study, the osteotomy line was modified upward in 3 models. However, translation did not occur prominently (Fig $5 \mathrm{~F})$. This is because the surface of the osteotomy included the level of the CORA on the tBL in all 3 models, and the angle to be corrected was relatively small.

OSCRO is a surgical method based on the coordination system of linear algebra (15). The combination of angular and torsional deformities can be represented by two cylinders. After dividing the proximal and distal parts by the CORA, the independent coordination system can be defined for each part. Then, the distal coordination system is rotated several times to match the proximal coordination system. Using the rotation matrix, it is possible to find a single axis of rotation. As such, the planning of OSCRO requires advanced mathematical knowledge. Therefore, in many reports, this is referred to as a common drawback. In the operation, the pin was placed parallel to each other. In order to achieve a precise rotation angle, the distal pin was bent for the angle to be rotated. Subsequently, the distal segment was rotated to achieve the parallel alignment of the pins. This method is dependent on a surgeon's skill and thereby possess a high risk of error. Thus various attempts such as $3 \mathrm{D}$ guides, special tools for planning, and 3d-Model rehearsal surgery have been adopted to achieve an accurate surgical outcome $(6,7,11,22)$.

The limitations of this study include the lack of consideration of soft tissue since it focused on bone malformation by using 3D models. Therefore, it could be difficult to reduce the bone segments perioperatively due to the tension of soft tissue. Additionally, improper restraint of patient's activity after surgery could affect bone healing and cause malunion.

\section{Conclusion}

OSCRO may be an effective surgical option for the treatment of MPL with femoral varus and torsional deformities. This approach has high surgical accuracy with clinically negligible unintended deformities. Despite the advanced techniques being used in OSCRO, the operating time was reduced compared to previous studies with other surgical practices. These findings support the use of OSCRO clinically in MPL with varus and torsional deformities, which may be necessary in small-breed dogs that commonly undergo distal femoral osteotomy for the treatment of patellar luxation.

\section{Acknowledgements}

This study was supported by research fund of Chungnam National University.

\section{References}

1. Adams RW, Gilleland B, Monibi F, Franklin SP. The effect 
of valgus and varus femoral osteotomies on measures of anteversion in the dog. Vet Comp Orthop Traumatol 2017; 30: 184-190.

2. Arthurs GI, Langley-Hobbs SJ. Complications associated with corrective surgery for patellar luxation in 109 dogs. Vet Surg 2006; 35: 559-566.

3. Brinker WO, Piermattei DL, Flo GL. The stifle joint. In: Piermattei DL, Flo GL, editors. Handbook of Small Animal Orthopaedics and Fracture Repair. Philadelphia, PA: Saunders. 1997: 516-580.

4. Brower BE, Kowaleski MP, Peruski AM, Pozzi A, Dyce J, Johnson KA, Boudrieau RJ. Distal femoral lateral closing wedge osteotomy as a component of comprehensive treatment of medial patellar luxation and distal femoral varus in dogs. Vet Comp Orthop Traumatol 2017; 30: 20-27.

5. Cashmore RG, Havlicek M, Perkins NR, James DR, Fearnside SM, Marchevsky AM, Black AP. Major complications and risk factors associated with surgical correction of congenital medial patellar luxation in 124 dogs. Vet Comp Orthop Traumatol 2014; 27: 263-270.

6. Dobbe JG, Pre KJ, Kloen P, Blankevoort L, Streekstra GJ. Computer-assisted and patient-specific 3-D planning and evaluation of a single-cut rotational osteotomy for complex long-bone deformities. Med Biol Eng Comput 2011; 49: $1363-1370$

7. Dobbe JGG, Du Pre KJ, Blankevoort L, Streekstra GJ, Kloen P. Computer-assisted oblique single-cut rotation osteotomy to reduce a multidirectional tibia deformity: case report. Strategies Trauma Limb Reconstr 2017; 12: 115-120.

8. Franklin SP, Dover RK, Andrade N, Rosselli D, Clark KM. Correction of antebrachial angulation-rotation deformities in dogs with oblique plane inclined osteotomies. Vet Surg 2017; 46: 1078-1085.

9. Gibbons SE, Macias C, Tonzing MA, Pinchbeck GL, McKee WM. Patellar luxation in 70 large breed dogs. J Small Anim Pract 2006; 47: 3-9.

10. Kim SY, Snowdon KA, DeCamp CE. Single oblique osteotomy for correction of antebrachial angular and torsional deformities in a dog. J Am Vet Med Assoc 2017; 251: 333-339.

11. Meyer DC, Siebenrock KA, Schiele B, Gerber C. A new methodology for the planning of single-cut corrective osteotomies of mal-aligned long bones. Clin Biomech (Bristol,
Avon) 2005; 20: 223-227.

12. Oxley B, Gemmill TJ, Pink J, Clarke S, Parry A, Baines S, Malcolm McKee W. Precision of a novel computed tomographic method for quantification of femoral varus in dogs and an assessment of the effect of femoral malpositioning. Vet Surg 2013; 42: 751-758.

13. Paley D. Rotation and angulation-rotation deformities. In: Principles of Deformity Corecction. Berlin, Heidelberg, New York: Springer. 2002, 235-275.

14. Roch SP, Gemmill TJ. Treatment of medial patellar luxation by femoral closing wedge ostectomy using a distal femoral plate in four dogs. J Small Anim Pract 2008; 49: 152-158.

15. Sangeorzan BJ, Sangeorzan BP, Hansen ST Jr, Judd RP. Mathematically directed single-cut osteotomy for correction of tibial malunion. J Orthop Trauma 1989; 3: 267-275.

16. Soparat C, Wangdee C, Chuthatep S, Kalpravidh M. Radiographic measurement for femoral varus in Pomeranian dogs with and without medial patellar luxation. Vet Comp Orthop Traumatol 2012; 25: 197-201.

17. Spencer A, Karen M. Stifle Joint. In: Tobias, $1^{\text {st }}$ ed. Missouri: Elsevier. 2012: 973-988.

18. Towle HA, Griffon DJ, Thomas MW, Siegel AM, Dunning D, Johnson A. Pre- and postoperative radiographic and computed tomographic evaluation of dogs with medial patellar luxation. Vet Surg 2005; 34: 265-272.

19. Troedhan A, Mahmoud Z, Wainwright M, Khamis M. Cutting bone with drills, burs, lasers and piezotomes: a comprehensive systematic review and recommendations for the clinician. Int J Oral Craniofac Sci 2017; 3: 20-33.

20. Wangdee C, Theyse LF, Techakumphu M, Soontornvipart K, Hazewinkel HA. Evaluation of surgical treatment of medial patellar luxation in Pomeranian dogs. Vet Comp Orthop Traumatol 2013; 26: 435-439.

21. Yasukawa S, Edamura K, Tanegashima K, Seki M, Teshima $\mathrm{K}$, Asano K, Nakayama T, Hayashi K. Evaluation of bone deformities of the femur, tibia, and patella in Toy Poodles with medial patellar luxation using computed tomography. Vet Comp Orthop Traumatol 2016; 29: 29-38.

22. Youngman J, Raptis D, Al-Dadah K, Monsell F. An accurate method of determining a single-plane osteotomy to correct a combined rotational and angular deformity. Strategies Trauma Limb Reconstr 2015; 10: 35-39. 